\title{
Prognostic markers in patients with cirrhosis and portal hypertension who have not bled
}

\author{
Maria Poca $^{\mathrm{a}}$, Angela Puente ${ }^{\mathrm{a}}$, Isabel Graupera ${ }^{\mathrm{a}}$ and Càndid Villanueva ${ }^{\mathrm{a}, \mathrm{b}, *}$ \\ ${ }^{a}$ Gastrointestinal Bleeding Unit. Department of Gastroenterology. Hospital de la Santa Creu i Sant Pau, \\ Autonomous University, Barcelona, Spain \\ ${ }^{\mathrm{b}}$ Centro de Investigación Biomédica en Red de Enfermedades Hepáticas y Digestivas (CIBEREHD o Ciberehd), \\ Barcelona, Spain
}

\begin{abstract}
Prognostic markers of compensated cirrhosis should mainly investigate factors involved with progression to decompensation because death in cirrhosis is related with decompensation. Portal hypertension plays a crucial role in the pathophysiology of most complications of cirrhosis. Accordingly, HVPG monitoring has strong prognostic value. An HVPG $\geqslant 10 \mathrm{mmHg}$ determines a significantly higher risk of developing decompensation. Esophageal varices also can develop when the HVPG is $\geqslant 10 \mathrm{mmHg}$, although an HVPG $\geqslant 12 \mathrm{mmHg}$ is required for variceal bleeding to occur. Monitoring the changes induced by the treatment of portal hypertension on HVPG, provides strong prognostic information. In compensated cirrhosis hemodynamic response is appropriate when the HVPG decreased to $<10 \mathrm{mmHg}$ or by $>10 \%$ from baseline, because the incidence of complications such as bleeding or ascites significantly decrease when these targets are achieved. Whether serum markers, such as the FibroTest, they, may be valuable to predict decompensation should be established. Transient Elastography is a promising technique that has shown an excellent accuracy to detect severe portal hypertension. However, whether it can adequately determine clinically significant portal hypertension, and risk of developing varices and decompensation, should be established. Magnetic Resonance Elastography is also promising.
\end{abstract}

Keywords: Compensated cirrhosis, Prognostic markers, hepatic venous pressure gradient, HVPG monitoring

\section{Introduction}

The natural history of cirrhosis involves an early asymptomatic stage or "compensated", which over time progresses to a symptomatic or "decompensated" stage, identified by the presence of complications of cirrhosis such as ascites, variceal bleeding or encephalopathy [1]. Furthermore, two distinct stages can be differentiated in the compensated phase of cirrhosis. The first stage is characterized by the absence of esophageal varices and is associated with a mortality of less than $1 \%$, while the second stage is characterized by the presence of varices and is associated with a mortality of $3.4 \%$ per year [1].

The course of cirrhosis progressively involves an increase in portal pressure, and worsening of hepatocellular function, which ultimately leads to the development of some of the complications defining the transi-

*Corresponding author: Càndid Villanueva, Gastroenterology Department, Hospital Santa Creu i Sant Pau, Mas Casanovas, 90. 08025 Barcelona, Spain. Tel.: +34 9355659 20; Fax: +34 9355656 08; E-mail: cvillanueva@santpau.cat. tion from the compensated to the decompensated phase of the disease [2]. A patient with compensated cirrhosis can have virtually the same survival and causes of death than general population, while a decompensated patient has a median survival of less than 2 years. Patients with compensated cirrhosis die mostly after transition to a decompensated stage, and the annual probability of moving from compensated to decompensated cirrhosis is around $15 \%$ of cases [1-3]. In keeping with this, prognostic markers of the compensated stage of cirrhosis should mainly investigate factors involved with progression to decompensation, because death in cirrhosis is clearly related to the development of decompensation. On the other hand, prognostic markers of the decompensated stage should also assess factors determining mortality.

\section{Prognostic assessment in pre-primary prophylaxis}

Portal hypertension, usually estimated by the hepatic venous pressure gradient or HVPG, plays a crucial role 
in the pathophysiology of most complications associated with cirrhosis [4]. Different studies have demonstrated the prognostic value of HVPG monitoring [5]. It has been shown that the risk of developing varices is significantly higher when the HVPG is $\geqslant 10 \mathrm{mmHg}$ and that patients with an HVPG $\geqslant 10 \mathrm{mmHg}$ have a significantly higher probability of developing decompensated cirrhosis [6,7]. The likelihood of decompensation, during a median of 4 years of follow-up, is $<$ $10 \%$ in patients with an HVPG $<10 \mathrm{mmHg}$ and is of $40 \%$ in patients with an HVPG $\geqslant 10 \mathrm{mmHg}$ [7]. In addition to the qualitative value of HVPG (presence or absence of an HVPG $\geqslant 10 \mathrm{~mm} \mathrm{Hg}$ ), the quantitative degree of portal hypertension is also relevant because it has been suggested that for each $1 \mathrm{mmHg}$ increase in HVPG there is an $11 \%$ higher risk of clinical decompensation [7].

The assessment of HVPG allows the distinction of two phases with different prognostic implications in compensated cirrhosis. There is an initial phase with moderate portal hypertension indicated by an HVPG > $6 \mathrm{mmHg}$ but $<10 \mathrm{mmHg}$ [6]. In this phase, patients have a very low risk of developing decompensation of cirrhosis. In a subsequent phase, portal hypertension progress to severe or clinically significant which is defined by an HVPG $\geqslant 10 \mathrm{mmHg}$ that determines a significantly higher risk of decompensation of cirrhosis [7, 8]. It has been observed that with disease progression, up to $41 \%$ of patients who initially had mild portal hypertension (with HVPG $<10 \mathrm{mmHg}$ ) develop clinically significant portal hypertension (with HVPG $\geqslant 10$ $\mathrm{mmHg}$ ) after 24 months of follow up [9].

Esophageal varices can develop when the HVPG is $\geqslant 10 \mathrm{mmHg}$ [8]. However, an HVPG $\geqslant 12 \mathrm{mmHg}$ is usually required for variceal bleeding to occur, and it has been demonstrated that the reduction of HVPG below this threshold value virtually abolishes the risk of bleeding [10].

Monitoring the changes induced by the treatment of portal hypertension on HVPG, provides strong prognostic information [5,11]. A relevant number of studies, mainly dealing with patients treated to prevent variceal rebleeding, have shown that the hemodynamic response is appropriate when the HVPG decreased to $<12 \mathrm{mmHg}$ or $>20 \%$ from to baseline $[8,12$, 13]. Patients who achieve these targets have a bleeding risk significantly reduced to below 10-15\% [14]. Hemodynamic response is also associated with a lower incidence of other complications of portal hypertension such as ascites, spontaneous bacterial peritonitis and hepatorenal syndrome, and with increased sur- vival [15-17]. However, recent studies suggest that the diagnostic accuracy of hemodynamic monitoring can be improved, mainly in compensated cirrhosis [14,18]. Although the negative predictive value of this technique is very high (around 90\%), positive predictive value is modest, especially in primary prophylaxis [19]. In primary prophylaxis the majority of patients (up to 60\%) do not have an HVPG reduction $\geqslant 20 \%$ from baseline with $\beta$-blockers, but despite it variceal bleeding occurs in few patients (around 15\%) [8,20,21]. A recent study shows that, in this setting, a cut-off reduction $>10 \%$ in HVPG can improve the diagnostic accuracy achieved using the cut-off of $20 \%$ decrease to define response, substantially increasing specificity [22]. In that study, by using the $10 \%$ target, the proportion of nonresponders decreased markedly, from $66 \%$ to $36 \%$, and a notable increase in the rate of first bleeding among nonresponders was also observed, from $27 \%$ to $46 \%$, thus improving the positive predictive value [22]. These results clearly suggest a relevant improvement in the diagnostic accuracy of HVPG monitoring in primary prophylaxis using the $10 \%$ target to define response.

In addition to the prognostic value for bleeding, monitoring of changes on HVPG also provides relevant prognostic information on the probability of developing ascites in patients with compensated cirrhosis at high risk of decompensation as indicated by a baseline HVPG $>10 \mathrm{mmHg}$ [7]. A recent study showed that once clinically significant portal hypertension has been reached, it is still possible to reduce the probability of developing ascites by achieving a decrease in HVPG $\geqslant 10 \%$ from baseline [23]. This represents a relevant issue because ascites is the most frequent complication of cirrhosis and is often the first decompensation to appear. On the other hand, that study showed that when such a reduction in HVPG was not achieved, ascites developed in the majority of patients (up to 90\%) [23]. Several studies have already suggested that, even in earlier stages of cirrhosis with compensated disease and moderate portal hypertension with HVPG $>6 \mathrm{mmHg}$ and absence of varices, a $10 \%$ threshold may be the best cutoff to predict the development of clinical decompensation [6,7].

Recent studies have also shown that the evaluation of acute response to administration of intravenous betablockers in a single hemodynamic study, can accurately predict the long-term outcome [22,24]. As compared to nonresponders, acute responders had a lower probability of a first bleeding, a lower risk of developing ascites during the follow-up and better survival [22]. This is a particularly important issue because assessing response 
in a single hemodynamic study, not only eliminates the need for a second invasive procedure, but also allows an immediate rescue therapy for nonresponders. It is possible that these factors will improve the diagnostic yield of hemodynamic monitoring and its applicability in clinical practice.

In addition to HVPG monitoring, different predictors of clinical decompensation related with the severity of portal hypertension and liver insufficiency have been identified. A recent study in compensated patients with hepatitis $C$ virus (both stage 1 and stage 2), identified the presence of esophageal varices and bilirubin as the only independent predictors of clinical decompensation [25]. Child-Pugh score and MELD score have been predictors in compensated patients in some studies $[25,26]$. In another study, in compensated patients with cirrhosis of different etiologies and portal hypertension, but without varices, HVPG, MELD, and albumin were independent predictors of the development of clinical decompensation [7]. However, HVPG had a predominant predictive role and was the most robust predictor of clinical decompensation, with a greater discriminative ability in the prediction of decompensation, which will ultimately determine survival.

Because disease-related complications of cirrhosis are mediated by the development and progression of hepatic fibrosis, a variety of serum markers have been developed for identifying patients who are at risk for clinically significant hepatic fibrosis. The practical advantages of serum fibrosis markers include their noninvasiveness, potential for widespread availability, and reproducibility. Among them, one of the most widely used and validated is the FibroTest [27,28]. Several independent and combined analyses have demonstrated excellent diagnostic performance for the detection of histological stage F4 fibrosis [27,28]. However, it is less accurate in detecting the presence of intermediate stages of fibrosis as compared to the detection of cirrhosis [28]. There is also a significant correlation between FibroTest values and HVPG values, but this correlation is weaker in patients with cirrhosis [27]. Although the FibroTest value was significantly higher in patients with severe portal hypertension, the area under the receiver operating characteristic curve for the diagnosis of severe portal hypertension was only 0.79 [27]. Thus, other studies are needed to confirm these results, especially in patients with compensated cirrhosis [29].

Other serum markers such as laminin levels or serum hyaluronic acid concentrations have shown acceptable positive predictive value for the prediction of severe portal hypertension, but poor negative predictive val- ue [30,31]. These markers cannot be currently used to detect the presence of severe portal hypertension [29].

Transient elastography has shown an excellent accuracy for detecting cirrhosis [32] with sensitivity and specificity values approaching $90 \%[33,34]$. For the detection of hepatic fibrosis between stages 2-4, however, its sensitivity and specificity are markedly reduced [33, 34]. Moreover, its accuracy is diminished when obesity and narrow rib interspaces are encountered [35]. Transient elastography has also shown an excellent accuracy for detecting severe portal hypertension with an HVPG above 10 to $12 \mathrm{mmHg}$ [36-38]. However, the correlation with HVPG was less strong in patients with an HVPG value above 10 or $12 \mathrm{~mm} \mathrm{Hg}$ [36-38]. Transient elastography has been more accurate than serum markers for detection of cirrhosis [39]. This suggests that it may be useful for determining the presence of clinically significant portal hypertension, with risk of developing esophageal varices and decompensation.

Additional large-scale prospective studies are needed to further define the role of Transient elastography for diagnosis and management of severe portal hypertension in compensated cirrhosis.

Magnetic resonance elastography has demonstrated feasibility and diagnostic accuracy in detecting hepatic fibrosis in initial prospective studies [40,41]. As with Transient elastography, the detection of cirrhosis by magnetic resonance elastography is highly accurate with sensitivity and specificity values exceeding $90 \%[40,41]$. This technique is not significantly affected by obesity or rib interspace width [39]. However, its correlation with the HVPG should be established.

\section{Prognostic assessment in primary prophylaxis}

Gastroesophageal varices are the most relevant portosystemic collaterals because their rupture results in variceal hemorrhage, the most common lethal complication of cirrhosis. Gastroesophageal varices are present in approximately $50 \%$ of patients with cirrhosis [42]. Their presence correlates with the severity of liver disease: while only $40 \%$ of Child A patients have varices, they are present in $85 \%$ of Child $\mathrm{C}$ patients $[42,43]$. Variceal hemorrhage occurs at a yearly rate of $5 \%-15 \%$, and the most important predictor of hemorrhage is the size of varices, with the highest risk of first hemorrhage (15\% per year) occurring in patients with large varices [44]. Other predictors of hemorrhage are decompensated cirrhosis (Child B/C) and the endoscopic presence of red wale marks [44]. 
In patients with cirrhosis the incidence of esophageal varices increases by nearly $5 \%$ per year, and the rate of progression from small to large varices is approximately 5 to $10 \%$ per year $[6,45,46]$. Practice guidelines recommend screening for varices in all patients with cirrhosis because nonselective beta-blockers and band ligation reduce the risk of first bleeding by $50 \%$ in patients with medium or large varices $[47,48]$. Prognostic markers in this setting should investigate factors indicating risk of varices (or large varices) and factors indicating risk of bleeding among those with varices.

\subsection{Detection of varices and prognostic markers for varices}

At present, upper gastrointestinal endoscopy is the gold standard for determining the presence of varices [47]. This technique is uncomfortable and invasive for patients and is costly and time-consuming, and it has been shown that there is interobserver variability as high as $30 \%$ for the detection of small esophageal varices. Moreover, since the point prevalence of medium/large varices is approximately $15 \%-25 \%$ [42], the majority of subjects undergoing screening endoscopy either do not have varices or have varices that do not require prophylactic therapy. Taking into account these issues, numerous non-invasive methods alternatives to endoscopy have been studied for determining the presence of varices, including biochemical and ultrasound methods, computed tomography (CT) scanning, video capsule endoscopy and spleen magnetic resonance imaging. Among them, video capsule endoscopy appears to be a very promising tool for the detection of esophageal varices. Video capsule endoscopy has been compared to conventional upper endoscopy in several studies [49-53]. A significant correlation has been found between capsule and standard endoscopy for the detection of varices. The sensitivity of capsule endoscopy ranged from $68 \%$ to $100 \%$ with a specificity ranging from $88 \%$ to $100 \%$ [49-53]. For the detection of large varices, the sensitivity was $78 \%$ with a specificity of $96 \%$ [52].

Some simple non-invasive methods may have a value as first line screening for esophageal varices. The diagnostic value of platelet count has been extensively studied, and a low platelet count has been repeatedly found to be related to the presence of esophageal varices $[39,46,54-56]$. However, the optimal threshold varies markedly from study to study, and its diagnostic value in patients with compensated cirrhosis has been questioned [57]. Some simple serum non-invasive markers such as the Lok index, the Forns' index or the platelet count/spleen diameter ratio have shown a good performance for diagnosis varices. Remarkably, in a recent large-scale multicenter study a combination of a Lok index (cutoff 1.5) and a Forns' index (cutoff 8.8) had a high negative predictive value for excluding presence of clinically relevant varices that would benefit from primary prophylaxis (large varices or small varices with red signs or in Child-Pugh class $\mathrm{C}$ patients) [58]. The platelet count/spleen diameter ratio is a promising index recently proposed, which has shown a $100 \%$ negative predictive value for excluding any grade varices [59]. However, the performance characteristics of the test in a following validation study were lower than in the original report [60]. Two small studies have also suggested that the FibroTest may be valuable for excluding presence of large varices $[39,61]$. Despite its excellent accuracy for detecting cirrhosis, transient elastography seems less sensitive for esophageal varices and did not perform better than serum indexes for both the detection of varices and large varices in a recent study [39].

Despite being very promising, at present, the predictive accuracy of such noninvasive markers is still unsatisfactory and additional large-scale prospective studies are needed to define the real role of these non-invasive markers for diagnosis and grading of varices. Until then these noninvasive markers should not dictate which patients should or should not be screened for esophageal varices by endoscopy $[47,48]$. On the other hand, it should be keep in mind that varices may appear when HVPG increases above $10 \mathrm{~mm} \mathrm{Hg} \mathrm{[6,8],} \mathrm{according-}$ ly patients with HVPG bellow $10 \mathrm{mmHg}$ do not need endoscopy to assess the development of varices.

\subsection{Prognostic markers of risk of bleeding in patients with varices}

Once developed, varices usually increase in size from small to large before they eventually rupture and bleed. The reported rate of progression is heterogeneous (5$30 \%$ per year) $[45,62,63]$. The factor that has been most consistently associated with variceal progression is liver failure, as assessed by Child-Pugh class [45,62,63]. Other factors include alcoholic aetiology of cirrhosis and presence of red wale markings at the varices [45, 62]. Changes in HVPG (either "spontaneous" or caused by therapy) are usually accompanied by parallel variations in the size of the oesophageal varices, which are significantly reduced when HVPG decreases below 12 mmHg [8]. 
In patients with cirrhosis the overall incidence of variceal bleeding is about $4 \%$ per year. This risk increases to $15 \%$ per year in patients with medium-large varices [64]. The most important predictive factors related to the risk of bleeding are variceal size, ChildPugh class and presence of red signs [44]. In addition, many studies have shown that variceal bleeding only occurs if the HVPG reaches a threshold value of $12 \mathrm{mmHg}[5,8,10]$. Varices do not develop until the HVPG increases up to $10 \mathrm{mmHg}$ [6], and it should be of at least $12 \mathrm{mmHg}$ for the appearance of variceal bleeding $[8,10]$. Longitudinal studies have demonstrated that if HVPG decreases below $12 \mathrm{mmHg}$ by means of treatment or spontaneously due to an improvement in liver disease, variceal bleeding is totally prevented and varices may decrease in size [8]. Even if this target is not achieved, a $20 \%$ decrease in portal pressure from baseline levels (or greater) also offers a marked protection from variceal bleeding $[5,12,13]$.

Patients with medium to large varices should be considered for prophylactic treatment of variceal bleeding $[47,48]$. It is well established that "small" varices with red signs or in Child-Pugh $\mathrm{C}$ class patients have a bleeding risk similar to that of large varices [44]. In keeping with this, it is currently recommended that these high risk varices should also receive prophylactic treatment $[47,48]$. Furthermore, $\beta$-blockers may reduce the rate of progression from small to large varices, and decrease the incidence of variceal bleeding in patients with small varices [63].

Longitudinal studies have demonstrated that a decrease in HVPG to $<12 \mathrm{mmHg}$ essentially eliminates the risk of bleeding and improves survival [8], while reductions $>20 \%$ from baseline or even $>10 \%$ from baseline significantly decrease the risk of first variceal bleeding [20-22], Whether or not HVPG monitoring may be useful to guide therapy is currently under investigation.

\section{Implications in future research in this area}

HVPG-monitoring provides important prognostic information that can help to improve the management of compensated cirrhosis offering different therapeutic targets. In a large multicenter study $\beta$-blockers were not useful to prevent the development of varices in compensated cirrhosis with early portal hypertension (HVPG $>5 \mathrm{mmHg}$ ) [6]. However, whether HVPGguided therapy may be useful to prevent decompensation of cirrhosis with clinically significant portal hy- pertension (HVPG $\geqslant 10 \mathrm{mmHg}$ ) at high risk of developing complications of portal hypertension should be investigated. Future studies should assess whether in these high-risk patients, $\beta$-blockers alone or combined with other drugs in hemodynamic non-responders to $\beta$-blockers may be useful to prevent decompensation.

In the genesis and maintenance of portal hypertension associated with cirrhosis, two mechanisms act synergistically [4]. Initially, there is an increased hepatic vascular resistance due to the disruption of liver architecture inherent to cirrhosis but also due, in part, to an increased hepatic vascular tone caused by the contraction of portal myofibroblasts and hepatic stellate cells [65]. With the progression of the disease, splanchnic vasodilatation develop and lead an increased splanchnic blood flow which maintains and aggravates portal hypertension [66]. $\beta$-blockers act on this second mechanism, decreasing portal pressure by a reduction of splanchnic blood flow as a result of drug-induced splanchnic vasoconstriction [67]. However, in early stages of portal hypertension (HVPG $<$ $10 \mathrm{mmHg}$ ), intrahepatic vasoconstriction may be the main mechanism that determines portal hypertension, while splanchnic vasodilatation may not yet be fully developed, which means less potential for vasoconstrictors [68]. This suggests that hepatic vasodilators, which may reduce portal pressure by decreasing intrahepatic vascular resistance, can be more effective in early stages of cirrhosis which should also be assessed in future studies.

Whether or not non-invasive methods to detect clinically significant portal hypertension may be useful in the management of patients with compensated cirrhosis, with or without varices, should also be investigated in future studies.

\section{References}

[1] G. D’Amico, G. Garcia-Tsao and L. Pagliaro, Natural history and prognostic indicators of survival in cirrhosis: A systematic review of 118 studies, J Hepatol 44 (2006), 217-231.

[2] G. Garcia-Tsao, S. Friedman, J. Iredale and M. Pinzani, Now there are many (stages) where before there was one: In search of pathophysiological classification of cirrhosis, Hepatology 51 (2010), 1445-1449.

[3] G. D'Amico, A. Morabito, L. Pagliaro and E. Marubini, Survival and prognostic indicators in compensated and decompensated cirrhosis, Dig Dis Sci 31 (1986), 468-475.

[4] G. Garcia-Tsao and J. Bosch, Management of varices and variceal hemorrhage in cirrhosis, N Engl J Med 362 (2010), $823-832$.

[5] G. D'Amico, J.C. Garcia-Pagan, A. Luca and J. Bosch, Hepatic vein pressure gradient reduction and prevention of variceal bleeding in cirrhosis: A systematic review, Gastroenterology 131 (2006), 1611-1624. 
[6] R.J. Groszmann, G. Garcia-Tsao, J. Bosch, N.D. Grace, A.K Burroughs, R. Planas, A. Escorsell, J.C. Garcia-Pagan, D. Patch, D.S. Matloff, H. Gao and R. Makuch, for the Portal Hypertension Collaborative Group. Beta-blockers to prevent gastroesophageal varices in patients with cirrhosis, $N$ Engl J Med 353 (2005), 2254-2261.

[7] C. Ripoll, R. Groszmann, G. Garcia-Tsao, N. Grace, A. Burroughs, R. Planas, A. Escorsell, J.C. Garcia-Pagan, R. Makuch, D. Patch, D.S. Matloff and J. Bosch, Hepatic venous pressure gradient predicts clinical descompensation in patients with compensated cirrhosis, Gastroenterology 133 (2007), 481-488.

[8] R.J. Groszmann, J. Bosch, N.D. Grace et al., Hemodynamic events in a prospective randomized trial of propranolol versus placebo in the prevention of a first variceal hemorrhage, Gastroenterology 99 (1990), 1401-1407.

[9] G. Garcia-Tsao, R.J. Groszmann, J. Bosch, N.D. Grace, A.K. Burroughs, R. Planas et al., Baseline hepatic venous pressure gradient (HVPG) and VHC infection predict the development of clinically significant portal hypertension (CSPH), Hepatology 48 (2008), 625A

[10] G. Garcia-Tsao, R.J. Groszmann, R.L. Fisher, H.O. Conn, C.E. Atterbury and M. Glickman, Portal pressure, presence of gastroesophageal varices and variceal bleeding, Hepatology 5 (1985), 419-424.

[11] G. Garcia-Tsao, J. Bosch and R.J. Groszmann, Portal hypertension and variceal bleeding- Unresolved issues. Summary of an american association for the study of liver diseases and european association for the study of liver single-topic conference, Hepatology 47 (2008), 1764-1772.

[12] F. Feu, J.C. García-Pagán, J. Bosch, A. Luca, J. Terés and A. Escorsell, Relation between portal pressure response to pharmacotherapy and risk of recurrent varcieal haemorrhage in patients with cirrosis, Lancet 346 (1995), 1056-1059.

[13] C. Villanueva, J. Balanzó, M.T. Novella, G. Soriano, S. Sainz, X. Torras et al., Nadolol plus isosorbide mononitrate compared with sclerotherapy for the prevention of variceal rebleeding, N Engl J Med 334 (1996), 1624-1629.

[14] U. Thalheimer, J. Bosch and A.K. Burroughs, How to prevent varices from bleeding: Shades of grey - The case for nonselective $\beta$-blockers, Gastroenterology 133 (2007), 2029-2036.

[15] J.G. Abraldes, I. Tarantino, J. Turnes, J.C. Garcia-Pagan, J. Rodés and J. Bosch, Hemodynamic response to pharmacological treatment of portal hypertension and long-term prognosis of cirrhosis, Hepatology 37 (2003), 902-908.

[16] C. Villanueva, J.M. López-Balaguer, C. Aracil, L. Kolle, B. González, J. Miñana, G. Soriano, C. Guarner and J. Balanzó, Maintenance of hemodynamic response to treatment for portal hypertension and influence on complications of cirrhosis, $J$ Hepatol 40 (2004), 757-765.

[17] C. Villanueva, J. Miñana, J. Ortix, A. Gallego, G. Soriano, $\mathrm{X}$. Torras et al., Endoscopic ligation compared with combined treatment with nadolol and isosorbide mononitrate to prevent recurrent variceal bleeding, N Engl J Med 345 (2001), 647655.

[18] U. Thalheimer, M. Mela, D. Patch and A.K. Burroughs, Targeting portal pressure measurements: A critical reappraisal, Hepatology 39 (2004), 286-290.

[19] C. Villanueva, C. Aracil, A. Colomo and J. Balanzo, Nonselective ?-blockers and hepatic venous pressure gradient monitoring: what lies behind nonresponse, Gastroenterology 134 (2008), 1626-1627.

[20] J. Turnes, J.C. Garcia-Pagan, J.G. Abraldes, M. HernandezGuerra, A. Dell'Era and J. Bosch, Pharmacological Reduc- tion of Portal Pressure and Long-Term Risk of First Variceal Bleeding in Patients with Cirrhosis, Am J Gastroenterol 101 (2006), 506-512.

[21] C. Merkel, M. Bolognesi, D. Sacerdoti, G. Bombonato, B. Bellini, R. Bighin and A. Gatta, The hemodynamic response to medical treatment of portal hypertension as a predictor of clinical effectiveness in the primary prophylaxis of variceal bleeding in cirrhosis, Hepatology 32 (2000), 930-934.

[22] C. Villanueva, C. Aracil, A. Colomo, V. Hernández-Gea, J.M. López-Balaguer, C. Alvarez-Urturi, X. Torras, J. Balanzó and C. Guarner, Acute hemodynamic response to beta-blockers and prediction of long-term outcome in primary prophylaxis of variceal bleeding, Gastroenterology 137 (2009), 119-128.

[23] V. Hernandez-Gea, A. Colomo, C. Aracil, M. Concepcion, M. Poca, X. Torras, H. Santos, C. Guarner and C. Villanueva, Relationship between changes in portal pressure and development of ascites in cirrhotic patients with severe portal hypertension, Hepatology 50 (2009), 313A.

[24] V. La Mura, J.G. Abraldes, J.C. Raffa, O. Retto, A. Berzigotti, J.C. García-Pagán and J. Bosch, Porgnostic value of acute hemodynamic response to i.v. propranolol in patients with cirrhosis and portal hypertension, J Hepatol 51 (2009), 279287.

[25] A. Sangiovanni, G.M. Prati, P. Fasani, G. Ronchi, R. Romeo, M. Manini, E. Del Ninno, A. Morabito and M. Colombo, The natural history of compensated cirrhosis due to hepatitis $\mathrm{C}$ virus: A 17-year cohort study of 214 patients, Hepatology (2006), 1303-1310.

[26] A.J. Sanyal, C. Banas, C. Sargeant, V.A. Luketic, R.K. Sterling, R.T. Stravitz, M.L. Shiffman, D. Heuman, A. Coterrell, R.A. Fisher, M.J. Contos and A.S. Mills, Similarities and differences in outcomes of cirrhosis due to nonalcoholic steatohepatitis and hepatitis C, Hepatology 43 (2006), 682-689.

[27] D. Thabut, F. Imbert-Bismut, D. Cazals-Hatem, D. Messous, M. Muntenaus, D.C. Valla et al., Relationship between the FibroTest and portal hypertension in patients with liver disease, Aliment Pharmacol Ther 26 (2007), 359-368.

[28] T. Poynard, R. Morra, P. Halfon, L. Castera, V. Ratziu, F. Imbert-Bismut et al., Meta-analyses of FibroTest diagnostic value in chronic liver disease, BMC Gastroenterol 7 (2007), 40 .

[29] D. Thabut, R. Moreau and D. Lebrec, Noninvasive Assessment of Portal Hypertension in Patients With Cirrhosis, Hepatology 53 (2011), 683-694.

[30] M. Kondo, S.J. Miszputen, M.M. Leite-Mor and E.R. Parise, The predictive value of serum laminin for the risk of variceal bleeding related to portal pressure levels, Hepatogastroenterology 42 (1995), 542-545.

[31] J. Kropf, A.M. Gressner and W. Tittor, Logistic-regression model for assessing portal hypertension by measuring hyaluronic acid (hyaluronan) and laminin in serum, Clin Chem 37 (1991), 30-35.

[32] L. Castera, X. Forms and A. Alberti, Non-invasive evaluation of liver fibrosis using transient elastography, J Hepatol 48 (2008), 835-847.

[33] J.A. Talwalkar, D.M. Kurtz, S.J. Schoenleber, C.P. West and V.M. Montori, Ultrasound-based transient elastography for the detection of hepatic fibrosis: systematic review and metaanalysis, Clin Gastroenterol Hepatol 5 (2007), 1214-1220.

[34] M. Friedrich-Rust, M.F. Ong, S.J. Martens, C. Sarrazin, J. Bojunga, S. Zeuzen et al., Performance of transient elastography for the staging of liver fibrosis: a meta-analysis, Gastroenterology 134 (2008), 960-974. 
[35] L. Castera, J. Foucher, P.H. Bernard, F. Carvalho, D. Allaix, W. Merrouche et al., Pitfalls of liver stiffness measurement: a 5-year prospective study of 13,369 examinations, Hepatology 51 (2010), 828-835.

[36] F. Vizzutti, U. Arena, R.G. Romanelli, L. Rega, M. Foschi, S. Colagrande et al., Liver stiffness measurement predicts severe portal hypertension in patients with HCV-related cirrhosis, Hepatology 45 (2007), 1290-1297.

[37] C. Bureau, S. Metivier, J.M. Peron, J. Selves, M.A. Robic, P.A. Gourraud et al., Transient elastography accurately predicts presence of significant portal hypertension in patients with chronic liver disease, Aliment Pharmacol Ther 27 (2008), 1261-1268.

[38] M. Lemoine, S. Katsahian, M. Ziol, P. Nahon, N. GanneCarrie, F. Kazemi et al., Liver stiffness measurement as predictive tool of clinically significant portal hypertension in patients with compensated hepatitis $\mathrm{C}$ virus or alcohol-related cirrhosis, Aliment Pharmacol Ther 28 (2008), 1102-1110.

[39] L. Castéra, B. Le Bail, F. Roudot-Thoraval, P.H. Bernard, J. Foucher, W. Merrouche et al., Early detection in routine clinical practice of cirrhosis and oesophageal varices in chronic hepatitis C: comparison of transient elastography (FibroScan) with standard laboratory tests and non-invasive scores, $J$ Hepatol 50 (2009), 59-68.

[40] M. Yin, J.A. Talwalkar, K.J. Glaser, A. Manduca, R.C. Grimm, P.J. Rossman et al., Assessment of hepatic fibrosis with magnetic resonance elastography, Clin Gastroenterol Hepatol 5 (2007), 1207-1213.

[41] L. Huwart, C. Sempoux, E. Vicaut, N. Salameh, L. Annet, E. Danse et al., Magnetic resonance elastography for the noninvasive staging of liver fibrosis, Gastroenterology 135 (2008), $32-40$.

[42] G. D'Amico, L. Pasta, S. Madonia, G. Tarantino, A. Mancuso, G. Malizia et al., The incidence of esophageal varices in cirrhosis, Gastroenterology 120 (2001), A2.

[43] G. D'Amico, Natural history of compensated cirrhosis and varices, in: Complications of Cirrhosis: Pathogenesis, Consequences and Therapy, T.D. Boyer and R.J. Groszmann, eds, American Association for the Study of Liver Diseases, 2001, pp. 118-123.

[44] The North Italian Endoscopic Club for the Study and Treatment of Esophageal Varices. Prediction of the first variceal hemorrhage in patients with cirrhosis of the liver and esophageal varices. A prospective multicenter study, $N$ Engl J Med 319 (1988), 983-989.

[45] M. Merli, G. Nicolini, S. Angeloni, V. Rinaldi, A. De Santis, C. Merkel et al., Incidence and natural history of small esophageal varices in cirrhotic patients, J Hepatol 38 (2003), 266-272.

[46] A.J. Sanyal, R.J. Fontana, A.M. DiBisceglie, J.E. Everhart, M.C. Doherty, G.T. Everson et al., The prevalence and risk factors associated with esophageal varices in subjects with hepatitis C and advanced fibrosis, Gastrointest Endosc 64 (2006), 855-864.

[47] G. Garcia-Tsao, A.J. Sanyal, N.D. Grace, W. Carey and the Practice Guidelines Committee of the American Association for the Study of Liver Diseases, the Practice Parameters Committee of the American College of Gastroenterology. Prevention and Management of Gastroesophageal Varices and Variceal Hemorrhage in Cirrhosis, Hepatology 46 (2007), 922-938.

[48] R. De Franchis, Revising consensus in portal hypertension: Report of the Baveno V consensus workshop on methodology of diagnosis and therapy in portal hypertension, J Hepatol $\mathbf{5 3}$ (2010), 762-768.
[49] G. Eisen, R. Eliakim, A. Zaman, J. Schwartz, D. Faigel, E. Rondonotti et al., The accuracy of PillCam ESO capsule endoscopy versus conventional upper endoscopy for the diagnosis of oesophageal varices: a prospective three-center pilot study, Endoscopy 38 (2006), 31-35.

[50] M.G. Lapalus, J. Dumortier, F. Fumex, S. Roman, M. Lot, B. Prost et al., Oesophageal capsule endoscopy versus oesophagogastroduodenoscopy for evaluating portal hypertension. A prospective comparative study of performance and tolerance, Endoscopy 38 (2006), 36-41.

[51] L.R. Pena, T. Cox, A.G. Koch and A. Bosch, Study comparing oesophageal capsule endoscopy versus EGD in the detection of varices, Dig Liver Dis 40 (2008), 216-223.

[52] R. de Franchis, G.M. Eisen, L. Laine, I. Fernandez-Urien, J.M. Herrerias, R.D. Brown et al., Esophageal capsule endoscopy for screening and surveillance of esophageal varices in patients with portal hypertension, Hepatology 47 (2008), 1595-1603.

[53] M.G. Lapalus, E. Ben Soussan, M. Gaudric, J.C. Saurin, P.N. D'Halluin, O. Favre et al., Esophageal capsule endoscopy vs. EGD for the evaluation of portal hypertension: a French prospective multicenter comparative study, Am J Gastroenterol 104 (2009), 1112-1118.

[54] A. Zaman, R. Hapke, K. Flora, H.R. Rosen and K. Benner, Factors predicting the presence of esophageal or gastric varices in patients with advanced liver disease, Am J Gastroenterol 94 (1999), 3292-3296.

[55] F. Schepis, C. Camma, D. Niceforo, A. Magnano, S. Pallio, M. Cinquegrani et al., Which patients with cirrhosis should undergo endoscopic screening for esophageal varices detection? Hepatology 33 (2001), 333-338.

[56] R. Madhotra, H.E. Mulcahy, I. Willner and A. Reuben, Prediction of esophageal varices in patients with cirrhosis, J Clin Gastroenterol 34 (2002), 81-85.

[57] A.A. Qamar, N.D. Grace, R.J. Groszmann, G. Garcia-Tsao, J. Bosch, A.K. Burroughs et al., Platelet count is not a predictor of the presence or development of gastroesophageal varices in cirrhosis, Hepatology 47 (2008), 153-159.

[58] G. Sebastiani, D. Tempesta, G. Fattovich, L. Castera, P. Halfon, M. Bourliere, F. Noventa, P. Angeli, A. Saggioro and A. Alberti, Prediction of oesophageal varices in hepatic cirrhosis by simple serum non-invasive markers: Results of a multicenter, large-scale study, J Hepatol 53 (2010), 630-638.

[59] E. Giannini, F. Botta, P. Borro, D. Risso, P. Romagnoli, A. Fasoli et al., Platelet count/spleen diameter ratio: proposal and validation of a non-invasive parameter to predict the presence of oesophageal varices in patients with liver cirrhosis, Gut $\mathbf{5 2}$ (2003), 1200-1205.

[60] E.G. Giannini, A. Zaman, A. Kreil, A. Floreani, P. Dulbecco, E. Testa et al., Platelet count/spleen diameter ratio for the noninvasive diagnosis of esophageal varices: results of a multicenter, prospective, validation study, Am J Gastroenterol 101 (2006), 2511-2519.

[61] D. Thabut, J.B. Trabut, J. Massard, M. Rudler, M. Muntenau, D. Messous et al., Non-invasive diagnosis of large oesophageal varices with FibroTest in patients with cirrhosis: a preliminary retrospective study, Liver Int 26 (2006), 271-278.

[62] M. Zoli, C. Merkel, D. Magalotti, C. Gueli, M. Grimaldi, A. Gatta et al., Natural history of cirrhotic patients with small esophageal varices: a prospective study, Am J Gastroenterol 95 (2000), 503-508.

[63] C. Merkel, R. Marin, P. Angeli, P. Zanella, M. Felder, E. Bernardinello et al., A placebo-controlled clinical trial of nadolol in the prophylaxis of growth of small esophageal varices in cirrhosis, Gastroenterology 127 (2004), 476-484. 
[64] G. D'Amico, L. Pagliaro and J. Bosch, Pharmacological treatment of portal hypertension: an evidence-based approach, Semin Liver Dis 19 (1999), 475-505.

[65] R. Wiest and R.J. Groszmann, The paradox of nitric oxide in cirrhosis and portal hypertension: Too much, not enough, Heptology 35 (2002), 478-491.

[66] Y. Iwakiri and R.J. Groszmann, The hyperdynamic circulation of chronic liver diseases: from the patient to the molecule, Hepatology 43(Suppl 1) (2006), S121-S131.
[67] J. Bosch, A. Berzigotti, J.C. Garcia-Pagan and J.G. Abraldes, The management ofn portal hypertension: Rational basis, available treatments and future options, J Hepatol 48 (2008), S68-S92.

[68] A.A. Qamar, R.J. Groszmann, N.D. Grace, G. Garcia-Tsao, A.K. Burroughs and J. Bosch, Lack of effect of non-selective beta-blockers on the hepatic venous pressure gradient (HVPG) in patients with mild portal hypertension: a tale of two studies, Hepatology 52(Suppl 1) (2010), 1020A. 


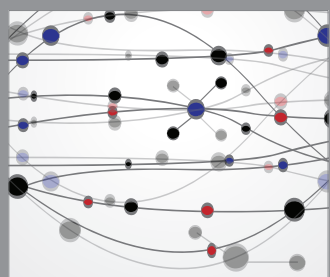

The Scientific World Journal
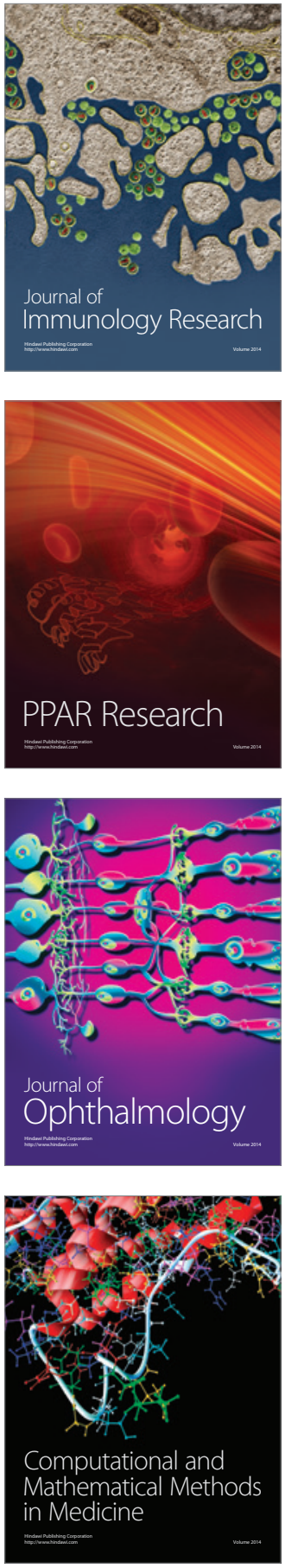

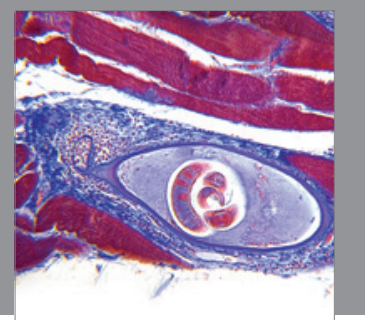

Gastroenterology

Research and Practice
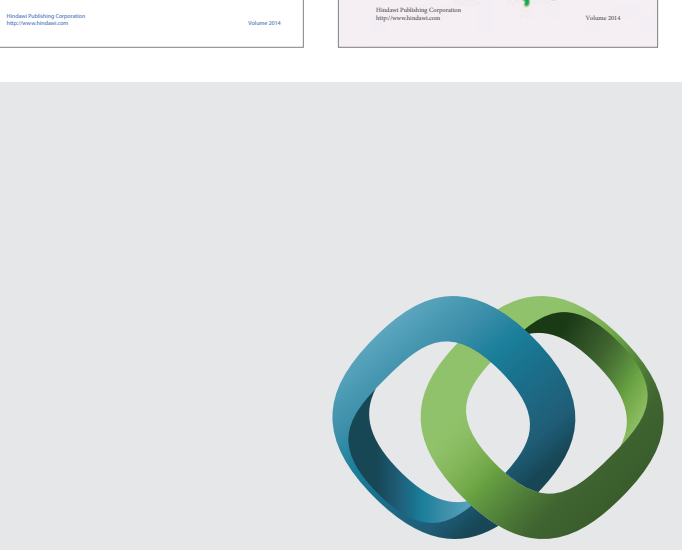

\section{Hindawi}

Submit your manuscripts at

http://www.hindawi.com
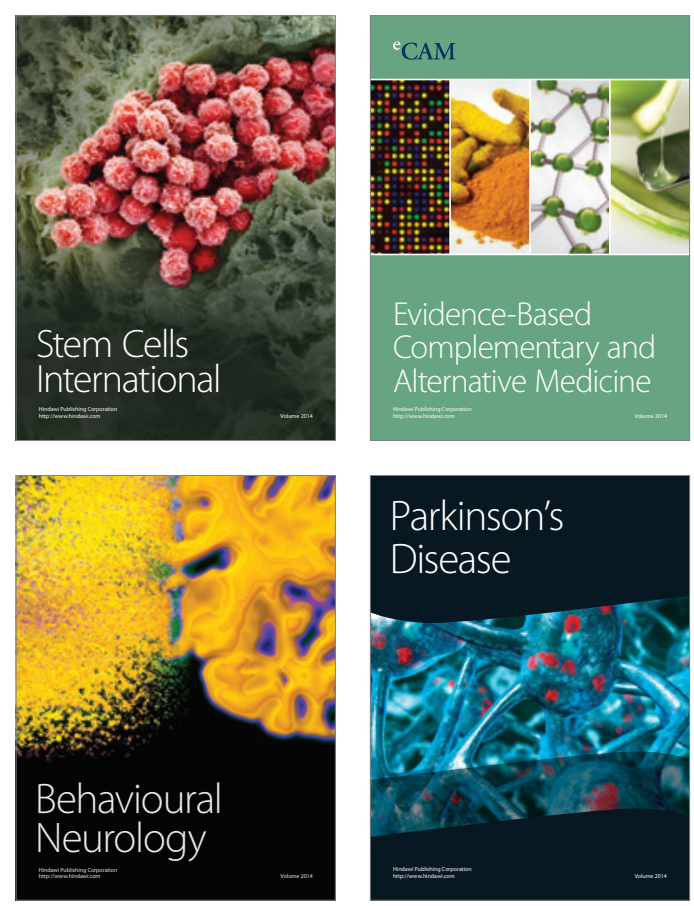

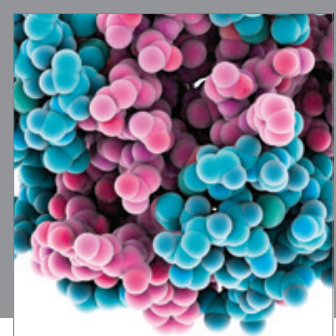

Journal of
Diabetes Research

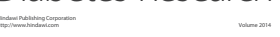

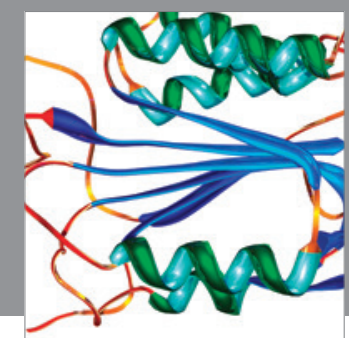

Disease Markers
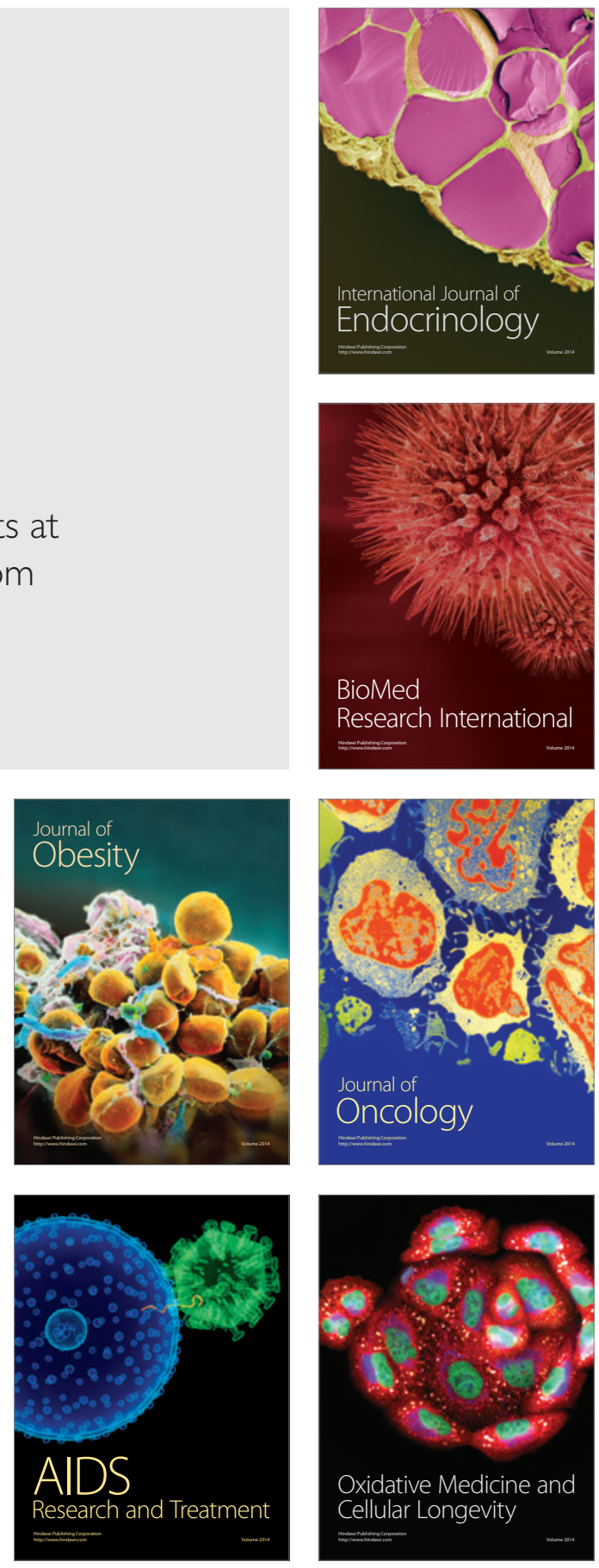